\title{
Bicuspid aortic valve annulus: assessment of geometry and size changes during the cardiac cycle as measured with a standardized method to define the annular plane
}

\author{
Sara Boccalini ${ }^{1}$ (D) - Lidia R. Bons ${ }^{2}$ - Allard T. van den Hoven ${ }^{2}$ - Annemien E. van den Bosch ${ }^{2}$ - Gabriel P. Krestin ${ }^{1}$. \\ Jolien Roos-Hesselink ${ }^{2}$. Ricardo P. J. Budde ${ }^{1,2}$
}

Received: 23 November 2020 / Revised: 11 February 2021 / Accepted: 19 March 2021 / Published online: 24 April 2021

(C) The Author(s) 2021

\begin{abstract}
Purpose Bicuspid aortic valve (BAV) is a complex malformation affecting not merely the aortic valve. However, little is known regarding the dynamic physiology of the aortic annulus in these patients and whether it is similar to tricuspid aortic valves (TAV). Determining the BAV annular plane is more challenging than for TAV. Our aim was to present a standardized methodology to determine BAV annulus and investigate its changes in shape and dimensions during the cardiac cycle.

Methods BAV patients were prospectively included and underwent an ECG-gated cardiac CTA. The annulus plane was manually identified on reconstructions at $5 \%$ intervals of the cardiac cycle with a new standardized method for different BAV types. Based on semi-automatically defined contours, maximum and minimum diameter, area, area-derived diameter, perimeter, asymmetry ratio (AR), and relative area were calculated. Differences of dynamic annular parameters were assessed also per BAV type.

Results Of the 55 patients included ( $38.4 \pm 13.3$ years; $58 \%$ males), 38 had BAV Sievers type 1 , 10 type 0 , and 7 type 2 . The minimum diameter, perimeter, area, and area-derived diameter were significantly higher in systole than in diastole with a relative change of $13.7 \%, 4.8 \%, 13.7 \%$, and $7.2 \%$ respectively (all $p<0.001$ ). The AR was $\geq 1.1$ in all phases, indicating an elliptic shape, with more pronounced flattening in diastole $(p<0.001)$. Different BAV types showed comparable dynamic changes.

Conclusions BAV annulus undergo significant changes in shape during the cardiac cycle with a wider area in systole and a more elliptic conformation in diastole regardless of valve type.

Key Points

- A refined method for the identification of the annulus plane on CT scans of patients with bicuspid aortic valves, tailored for the specific anatomy of each valve type, is proposed.

- The annulus of patients with bicuspid aortic valves undergoes significant changes during the cardiac cycle with a wider area and more circular shape in systole regardless of valve type.

- As compared to previously published data, the bicuspid aortic valve annulus has physiological dynamics similar to that encountered in tricuspid valves but with overall larger dimensions.
\end{abstract}

Keywords Bicuspid aortic valve · Cross-sectional anatomy · Cardiovascular physiological phenomena $\cdot$ Computed tomography angiography $\cdot$ Transcatheter aortic valve replacement

Sara Boccalini

sara.boccalini@yahoo.com

1 Department of Radiology, Erasmus Medical Center, P.O. Box 2040, 3000, CA Rotterdam, The Netherlands

2 Department of Cardiology, Erasmus Medical Center, P.O. Box 2040, 3000, CA Rotterdam, The Netherlands

$\begin{array}{ll}\text { Abbreviations } \\ \text { AR } & \text { Asymmetry ratio } \\ \text { BAV } & \text { Bicuspid aortic valve } \\ \text { LCC } & \text { Left coronary cusp } \\ \text { LVOT } & \text { Left ventricular outflow tract } \\ \text { NCC } & \text { Non-coronary cusp } \\ \text { RA } & \text { Relative area } \\ \text { RCC } & \text { Right coronary cusp }\end{array}$

Abbreviations

AR Asymmetry ratio

BAV Bicuspid aortic valve

LCC Left coronary cusp

LVOT Left ventricular outflow tract

NCC Non-coronary cusp

RCC Right coronary cusp 
TAV Tricuspid aortic valve

TAVI Transcatheter aortic valve implantation

VR Volume rendering

\section{Introduction}

With an estimated incidence of $0.4-2 \%$ in the general population, bicuspid aortic valve (BAV) is likely the most common congenital cardiac anomaly [1-4]. BAV are prone to precocious stenosis and regurgitation, often requiring an intervention early in life [5]. Nevertheless, this disease does not affect exclusively the valve but the entire valvular apparatus and aorta resulting in a complex disease. In addition, these structures are exposed to continuous and intense forces during the cardiac cycle resulting in arduous analysis and classification.

For instance, the annulus has been shown to have an elliptical shape that undergoes conformational changes during the cardiac cycle in a normal as well as stenotic tricuspid aortic valve (TAV) using ECG-gated CT imaging [6, 7]. Furthermore, most sizing parameters for the TAV aortic annulus show significant changes between diastole and systole [8]. If, and to what extent, such dynamic geometry changes also occur in the BAV annulus is unknown.

The broad variability in cusp morphology of BAV results in the cumbersome and, more importantly, sometimes impossible identification of the annulus plane as defined based on the standard anatomy of TAV. Therefore, a specific and standardized method for the definition of the annulus plane in BAV patients is strongly needed but lacking.

Comprehension of the physiology is likely to lead to adapted and specific pre-procedural evaluation and improved therapeutic strategies of BAV patients. The distortion of the valve apparatus in BAV patients has led to the exclusion of patients with this anomaly from earliest studies assessing safety and results of transcatheter aortic valve implantation (TAVI), due to concerns about deformation and malposition of the implanted valve [9-11]. Yet, in the last few years, the procedure has been performed with an increasing frequency in BAV patients. In fact, although with limitations due to the inclusion of small populations, later investigations have shown that TAVI is in fact feasible also in the BAV population and, furthermore, has results comparable to those obtained in cohorts with TAV [12-15].

Based on a revised method to determine the annulus plane, we assessed the presence and magnitude of geometrical and dimensional changes of the annulus during the cardiac cycle using ECG-gated CT as well as investigated if different BAV subtypes present different patterns.

\section{Materials and methods}

\section{Study population and BAV classification}

Sixty consecutive patients with a BAV were prospectively included as part of a clinical study at the Erasmus Medical Center, Rotterdam, The Netherlands, between November 2014 and March 2016 as previously reported [16, 17]. The local medical ethics committee approved the study and all patients provided written informed consent. As part of the study, an ECG-gated contrast-enhanced CT of the heart was performed.

The morphology of the aortic valve was classified as previously described by Sievers et al [1] based on images obtained with echo, CT, and magnetic resonance.

\section{CT scans}

Contrast-enhanced CT scans were acquired with a thirdgeneration dual-source CT scanner (Somatom Force, Siemens Healthineers) with the parameters summarized in Supplemental Material Table I. All scans were performed with retrospective ECG gating, $192 \times 0.6 \mathrm{~mm}$ collimation and $250 \mathrm{~ms}$ rotation time. Reconstructions of $1.5 \mathrm{~mm}$ thickness were performed throughout the entire cardiac cycle at 5\% intervals resulting in $20 \mathrm{im}-$ age datasets per patient.

\section{Annulus analysis}

A radiologist with $>5$ years of experience in cardiovascular radiology (S.B.) defined the annulus plane and semiautomatically measured the annulus dimensions on the CT scans for each of the 20 reconstructed phases.

The beginning of the systole was identified as the first phase where the aortic valve was opening/was open and its end as the first phase where the valve was closed.

\section{Annulus plane definition}

Images were exported to a commercially available workstation (IntelliSpace Portal, Philips) to allow for manual multiplanar reconstructions (MPR). The annulus plane was identified with a different methodology depending on the morphology of the bicuspid valve. In BAV with three equally/similarly sized cusps (Sievers type 1 and 2), the annulus plane was identified as the plane passing through the three hinge points (defined as the lowest insertion point of the valve leaflet on the aortic wall) as indicated by guidelines for TAV [18]. In case the fused cusps had different dimensions (Sievers type 1) or there were only two cusps (Sievers type 0 ), the annulus plane was identified with a newly 
Fig. 1 Determination of the annulus in patients with BAV type 1 with asymmetric cusps. (a) Volume rendered (VR) reconstructions in systole (a1) and diastole (a2) demonstrating the asymmetry of the sinuses due to the smaller dimensions of the right coronary cusp (RCC) (arrows). (b-d) Wrong annulus plane definition based on the three hinge points. In (b1) and (c1) schematic representations of the LVOT, sinuses of Valsalva (red, yellow, blue lines) and

sinotubular junction (gray line) where the annulus plane (green line) was identified as the plane passing through the three hinge points (hinge point of the RCC in red; hinge point of the noncoronary cusp (NCC) in blue; hinge point of the left coronary cusp (LCC) in yellow). In (b2b3) and (c2-c3), VR reconstructions showing the angulation between the centerline passing through the LVOT/aortic root and the line (b2-b3 and c2-c3; green lines) passing through the hinge points of the LCC and the NCC (b) and RCC (c), respectively. In (d1) and (d2), MPR showing the position of the axis in the longitudinal planes when identifying the plane passing through the three hinge points (d3). (e-f) Correct annulus plane definition. At first, one plane passing through the two hinge points of the two biggest cusps is identified (e1); then, this plane is tilted along the only still undetermined direction (e2) until the minimum crosssectional area and/or a plane perpendicular to the centerline is obtained (e3). In (f1) and (f2), MPR showing the position of the axis in the longitudinal planes when determining the plane passing through the two hinge points and then make adjustments by tilting the violet and orange axis to identify the smallest possible area and a plane perpendicular to the centerline (e3)
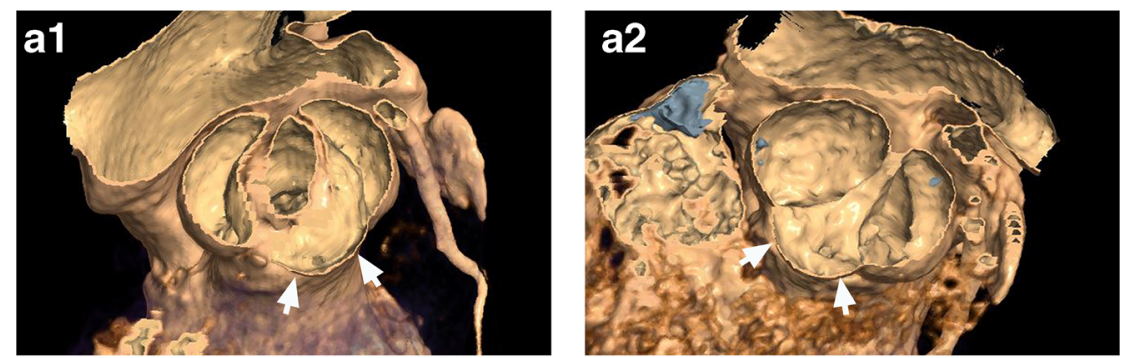

b1

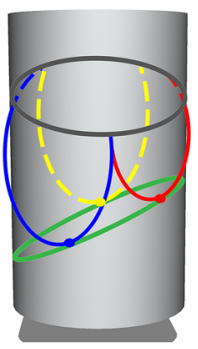

c1
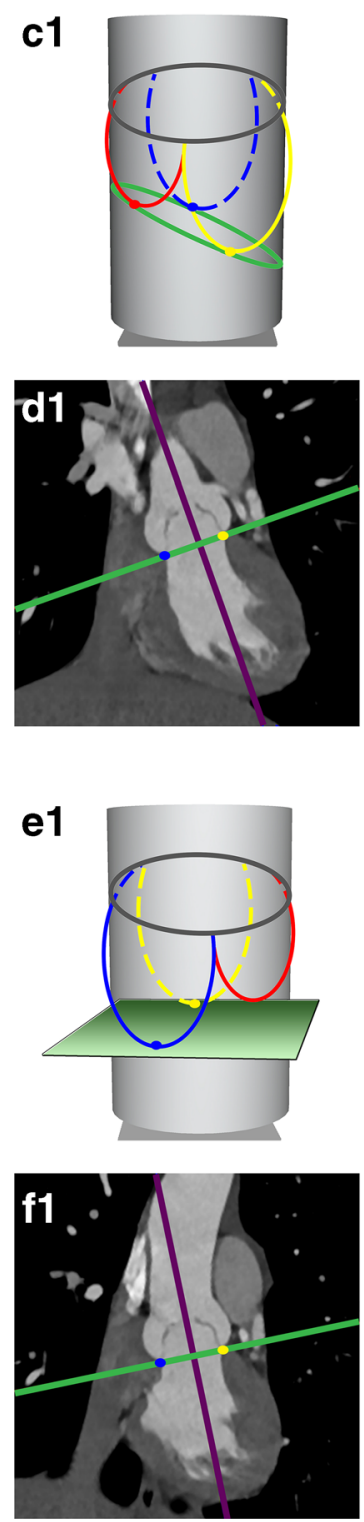
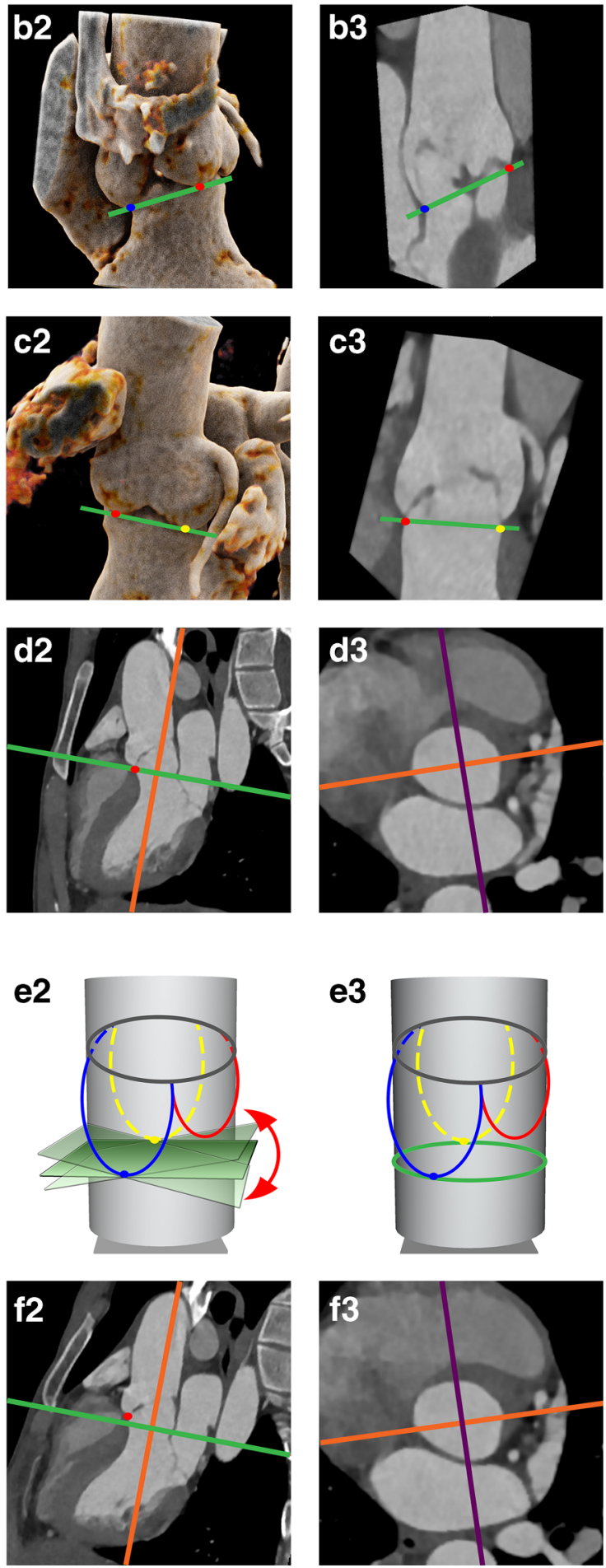

e3
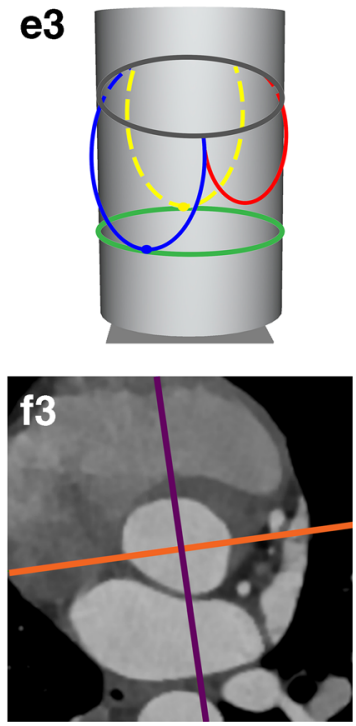
Fig. 2 Determination of the annulus plane in patients with BAV type 0. (a) VR reconstructions in systole (a1) and diastole (a2) showing the presence of only two cusps. (b) 3D anatomy of the aortic root. (c-d) One plane passing through the two hinge points of the two cusps is defined (c1); then, this plane is tilted along the only still undetermined direction (c2) until the minimum crosssectional area and/or a plane perpendicular to the centerline is obtained (c3). In (d1) and (d2), MPR showing the position of the axis in the longitudinal planes when determining the annulus plane (d3) as detailed above
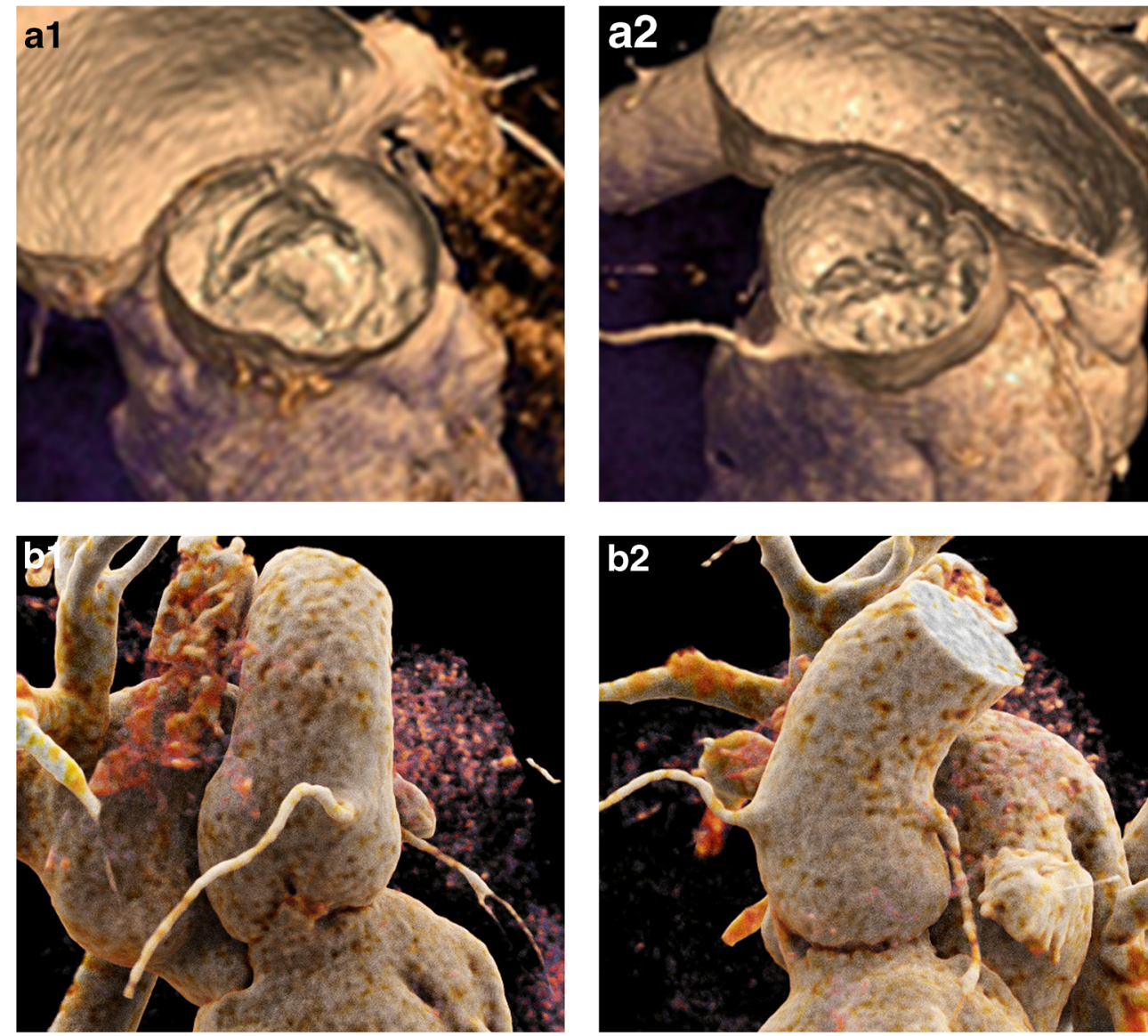

c1
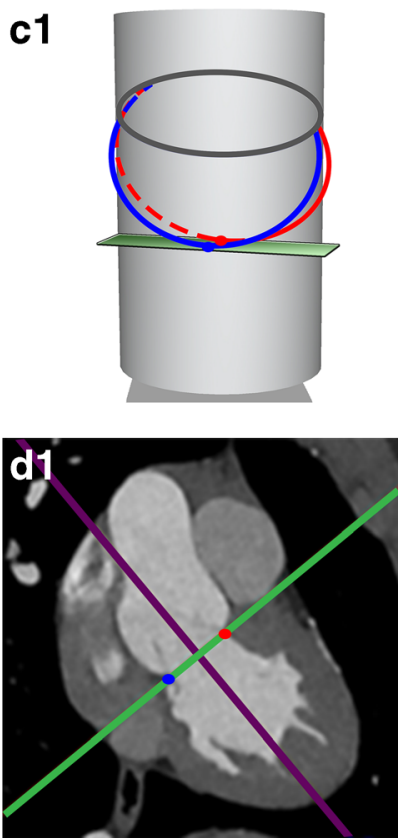

c2
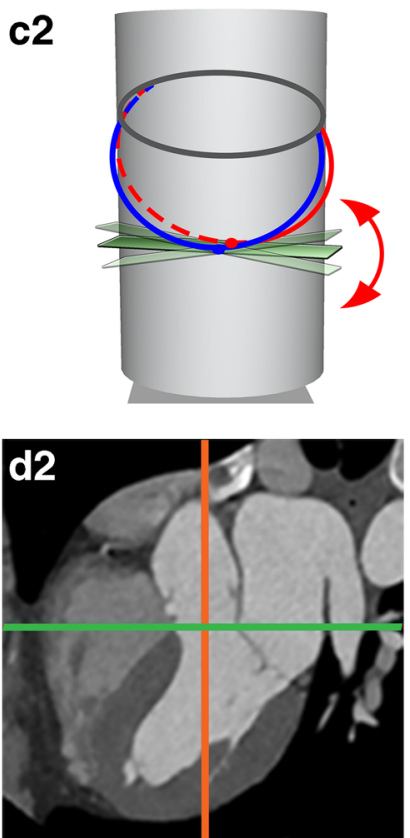

c3
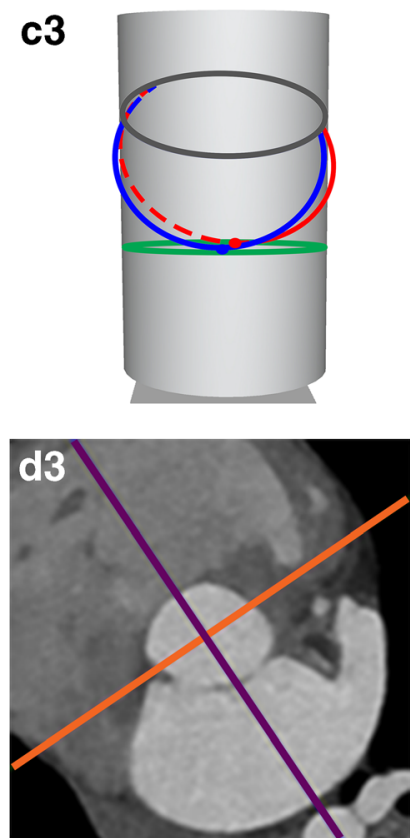

developed approach consisting of multiple steps based on a combination of previously published definitions of the annulus: first, one of the planes passing through the hinge point of the non-fused cusp and the hinge point of the bigger one of the two fused cusps (or through the two hinge points in valves type 0) was identified; then, this plane was tilted until the minimum annulus area at that level was identified [19-22]; if this 
plane was considered to be too steeply angled in respect to the LVOT and the sinuses, a compromise between the plane with the smallest diameter and a plane perpendicular to the centerline at this level was reached [23, 24] (Figs. 1 and 2). The plane was checked and adapted when necessary for each of the analyzed phases to account for displacement of the annulus due to the contraction of the heart.

\section{Annulus measurements}

The annulus measurements were performed semiautomatically with the "edge finder" tool available in the reconstruction software (IntelliSpace Portal). With this tool, two seed points are placed somewhere inside the annulus and in the tissues surrounding the aortic root, respectively. Thereafter, the software automatically defines the contour of the annulus where there is a sharp transition of Hounsfield unit values between the contrast-enhanced lumen and surrounding tissues. For each phase, the automatically traced contour was checked and adjusted when deemed necessary. The contour was modified to encircle the inner edge of small calcifications and to extend over big calcifications in continuity with the adjacent outline. The maximum diameter, the diameter perpendicular to the maximum (referred to as "the minimum diameter"), the area, and the perimeter were recorded (Fig. 3). The average diameter was calculated as the average of the maximum and minimum. The effective diameter was derived from the area [18]. The asymmetry ratio (AR) and relative area (RA) were calculated as previously defined [6]. The AR was defined as the ratio between the maximum and the minimum diameter. The RA was calculated as the ratio between the area at each individual phase and the average area of all phases throughout the cycle.
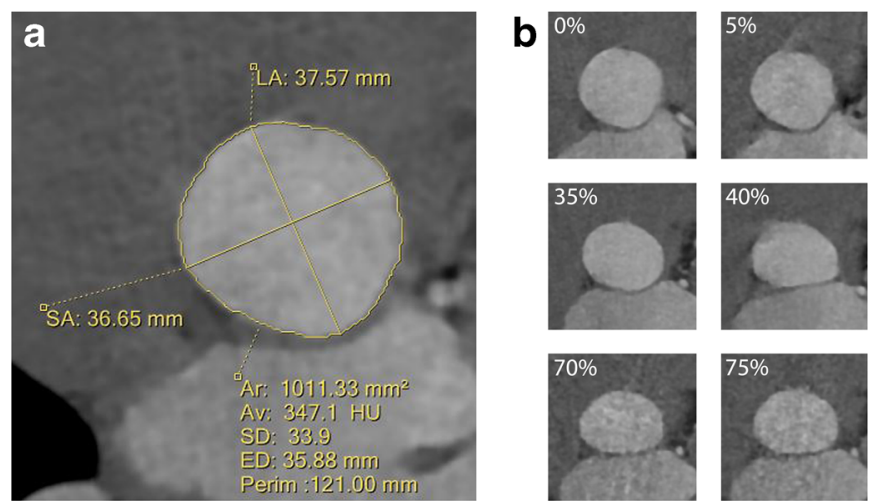

Fig. 3 Annulus measurements. (a) Measurement of the annulus parameters (maximum and minimum diameters, perimeter, area, and areaderived diameter) based on the semi-automatically defined contour. (b)
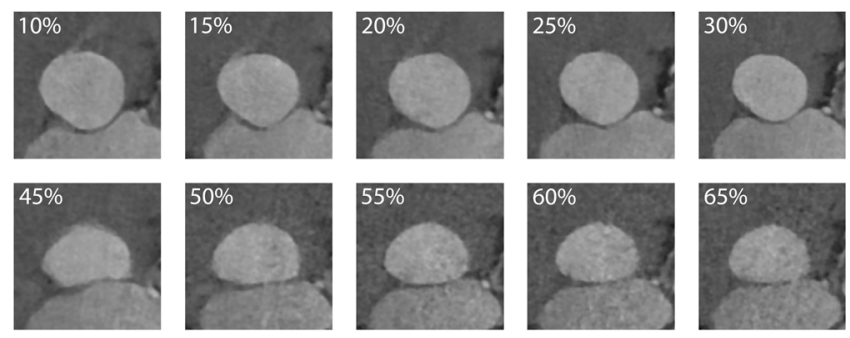

\section{Image quality assessment}

The image quality of each phase at the level of the annulus was subjectively assessed based on a 2-point scale (insufficient quality; acceptable to perfect quality) based on the following criteria: the definition of the cusps and hinge points and subsequent reliability in motion artifacts; the adequate opacification of the LVOT and aortic root. The phases with insufficient quality were excluded from further analysis.

The same observer re-measured all phases of one-third of the patients $(n=18)$ at least 1 month after the first measurement. imaging (L.B.) blinded to previous results analyzed 20 phases of 6 patients.
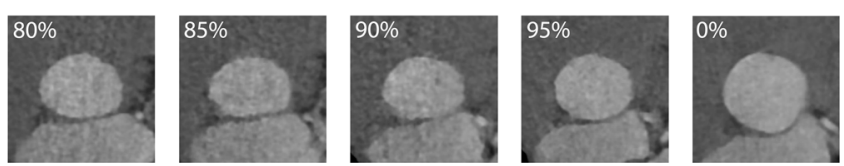

A case example showing all the 20 phases analyzed in a patient with BAV type $1 \mathrm{LR}$. The annulus shows a more circular shape in systole and a more elliptic shape during diastole 


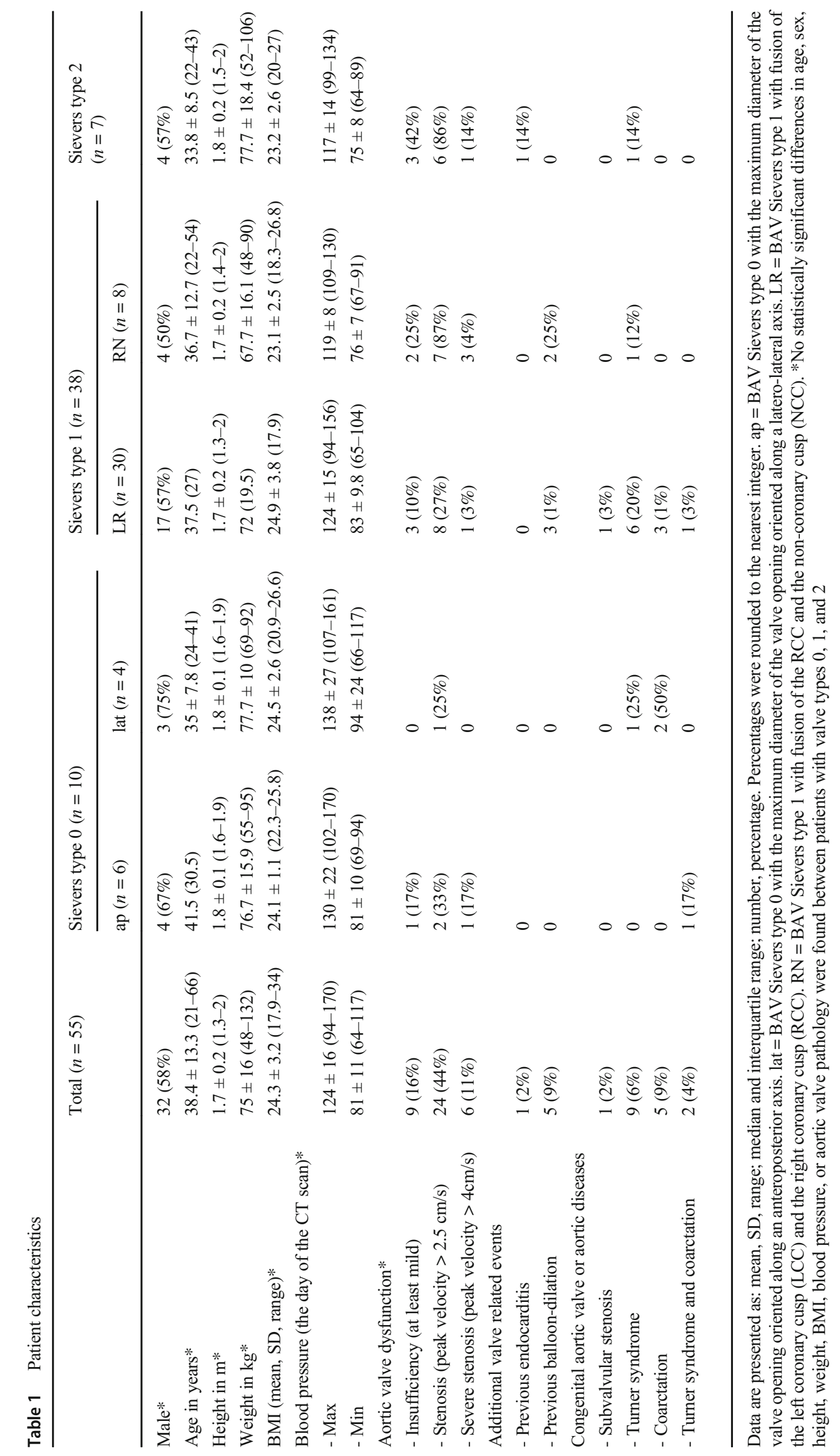


Table 2 Changes of the annulus parameters values during the cardiac cycle

\begin{tabular}{lcccc}
\hline & Max value* & Min value* & $\begin{array}{l}\text { Relative difference }[\%] \dagger \neq \\
( \pm \text { SD of the difference })\end{array}$ & $\begin{array}{l}p \text { value } \\
\text { Min vs Max }\end{array}$ \\
\hline Maximum diameter [mm] & $30.2 \pm 4.5$ & $28.4 \pm 4.2$ & $3.6 \%( \pm 3.9 \%)$ & $<0.001$ \\
Minimum diameter [mm] & $22.4 \pm 4.1$ & $26.0 \pm 4.1$ & $13.8 \%( \pm 8.4 \%)$ & $<0.001$ \\
Average diameter [mm] & $27.8 \pm 4.1$ & $25.4 \pm 4$ & $6.7 \%( \pm 3.5 \%)$ & $<0.001$ \\
Perimeter [mm] & $92.9 \pm 13.7$ & $86.9 \pm 14$ & $4.8 \%( \pm 4.3 \%)$ & $<0.001$ \\
Area [mm $\left.{ }^{2}\right]$ & $587 \pm 169$ & $487 \pm 144$ & $13.7 \%( \pm 6.8 \%)$ & $<0.001$ \\
Diameter based on area [mm] & $27.1 \pm 4.0$ & $24.6 \pm 3.7$ & $7.2 \%( \pm 3.7 \%)$ & $<0.001$ \\
Relative area & $1.1 \pm 0.1$ & $0.9 \pm 0.1$ & $13.8 \%( \pm 8.3 \%)$ & $<0.001$ \\
Asymmetry ratio & $1.3 \pm 0.2$ & $1.1 \pm 0.1$ & $12.8 \%( \pm 10.8 \%)$ & $<0.001$ \\
\hline
\end{tabular}

*Values calculated based on all data available for each phase; ${ }^{\dagger}$ relative difference compared to the maximum value; ${ }^{\ddagger}$ values calculated based on the number of cases available per comparison of the means; values for relative area and asymmetry ratio were calculated with Wilcoxon signed-ranked test

\section{Results}

\section{Patients and valve characteristics}

Of the 60 eligible patients, 5 were excluded because of overall insufficient image quality $(n=1)$, not all reconstruction phases available $(n=3)$, and the presence of a large septal aneurysm $(n=1)$.

In total, 55 patients were included (mean age: $38.4 \pm 13.3$ (21-66); males: $32(58 \%)$ ) of whom 10 had a Sievers type 0 BAV, 38 a type 1 BAV, and 7 a type 2 valve. Patients' characteristics are summarized in Table 1.

In 9 patients with Sievers type 1, the new method for the definition of the annulus plane based on two hinge points was used. Ten patients had calcifications of the annulus that were of considerable amount in only one case.

\section{Variation of annulus parameters during the cardiac cycle}

Out of a total of 1100 phase datasets (55 patients with 20 reconstructed phases each), $71(6.4 \%)$ were excluded due to insufficient quality. The excluded datasets were randomly distributed over the cardiac cycle $(p=0.6)$.

All of the measured annulus parameters presented statistically significant differences during the cardiac cycle (Table 2 and Fig. 4). The minimum diameter, the average diameter, the area, the perimeter, and the area-derived perimeter demonstrated the highest values during early systole $(5-10 \%$ of the cardiac cycle) with a significant reduction in diastole of $13.7 \%, 6.7 \%, 13.7 \%, 4.8 \%$, and $7.2 \%$, respectively (all $p$ values $<0.001$ ). The RA was $>$ 1 during systole $(0-30 \%)$ and in late diastole (90-100\%) $(p<0.001)$. The maximum diameter showed the highest value in diastole $(p<0.001)$. The AR was $\geq 1.1$ in all phases indicating an elliptic shape of the annulus throughout the entire cardiac cycle. The annulus demonstrated significant deformation with increased elliptic morphology during diastole (relative change of $13.9 \%$; $p$ $<0.001)$.

\section{Variation of annulus parameters during the cardiac cycle per type of BAV}

When analyzed separately, all three types of BAV showed analogous dynamic changes (Table 3 and Fig. 5). All assessed parameters showed significant differences over time, with the exceptions of the maximum diameter for type 2 valves $(p=0.15)$ and maximum diameter and perimeter for type 0 valves $(p=0.2$ and $p=0.2$ respectively) that did not show significant differences.

The AR was significantly different between BAV types 1 and 2 in early systole with valves type 1 showing a more elliptic annulus $(p=0.024$ at $10 \% ; p=0.014$ at $15 \% ; p=$ 0.023 at $20 \% ; p=0.016$ at $25 \%$ ). Patients with valves type 0 demonstrated a significantly more elliptic annulus than type 2 at phase $10 \%(p=0.047)$. The other parameters did not show significant differences between valve types at any of the time points.

\section{Influence of heart rate on annulus dynamics}

Scans were divided in 7 different categories based on heart rate (Table 4). All groups showed a smaller area between 40 and $60 \%$ of the cardiac cycle (Fig. 6); however, the smallest values were reached earlier in the group with the slowest heart rate.

\section{Intra- and inter-observer variability}

Intra- and inter-observer variabilities were good with all mean differences of $\leq 0.5 \pm 0.6 \mathrm{~mm}$ and $\leq 1 \pm 1.2 \mathrm{~mm}$ for maximum 
a

Maximum diameter

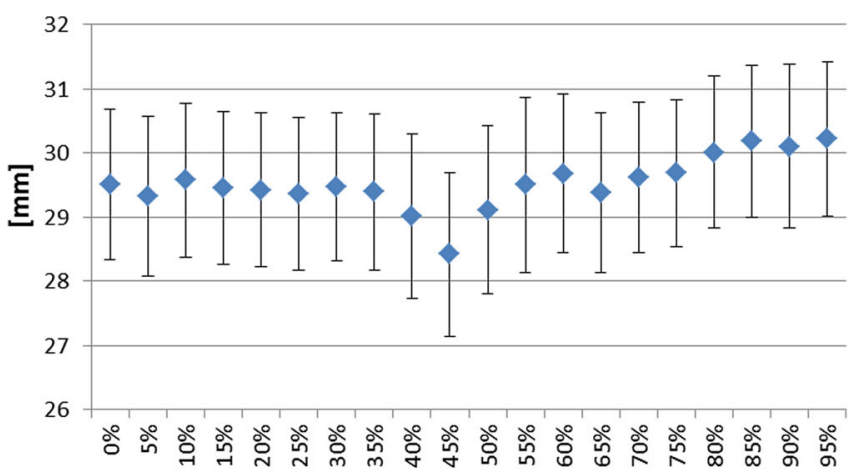

C

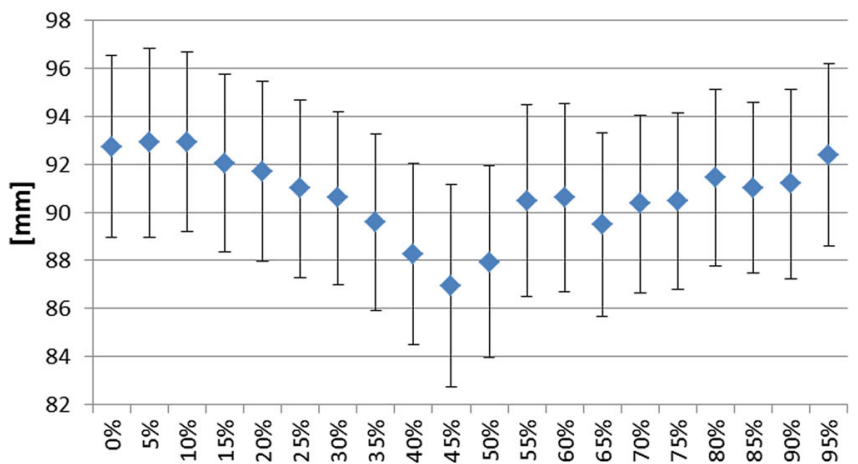

e

Average diameter

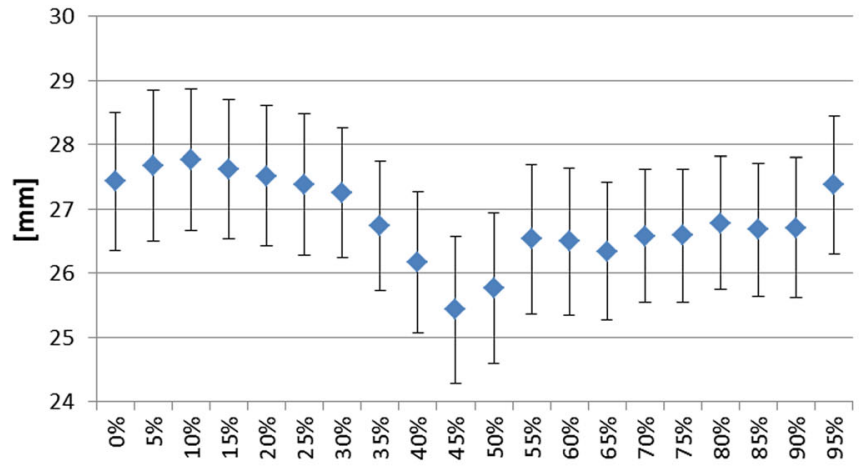

g

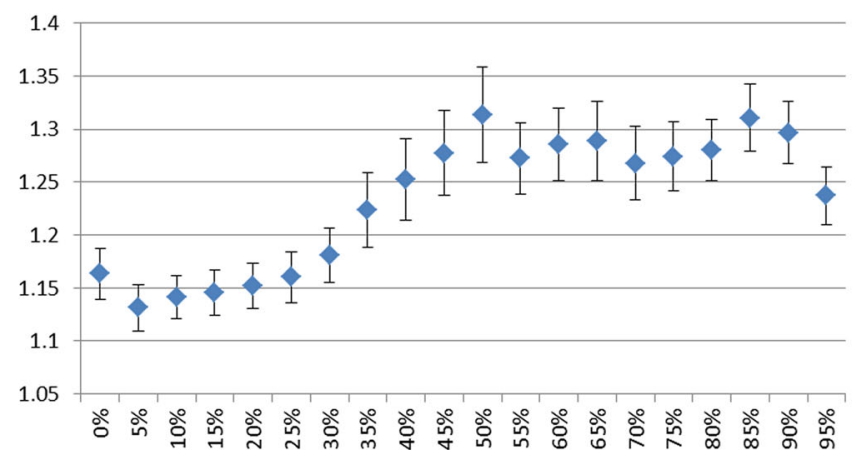

b

Minimum diameter

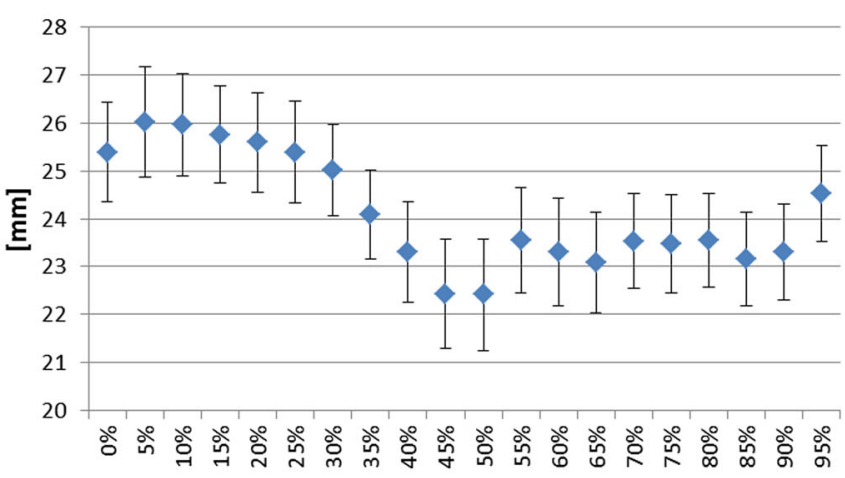

d Area

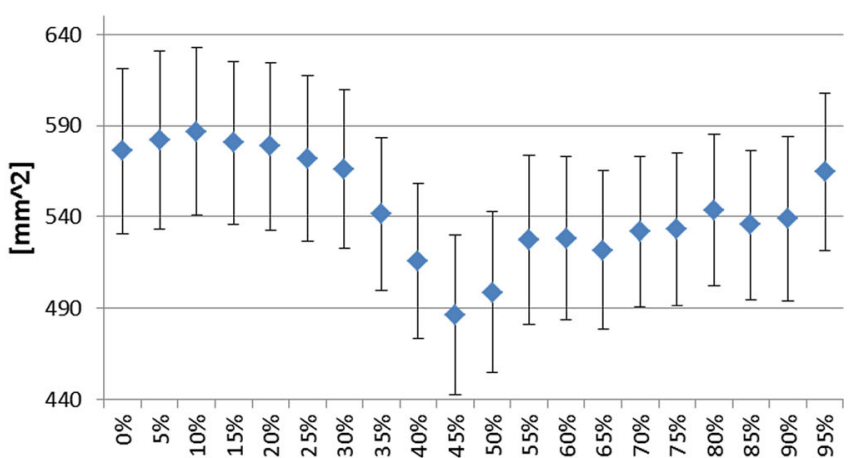

f

Area derived diameter

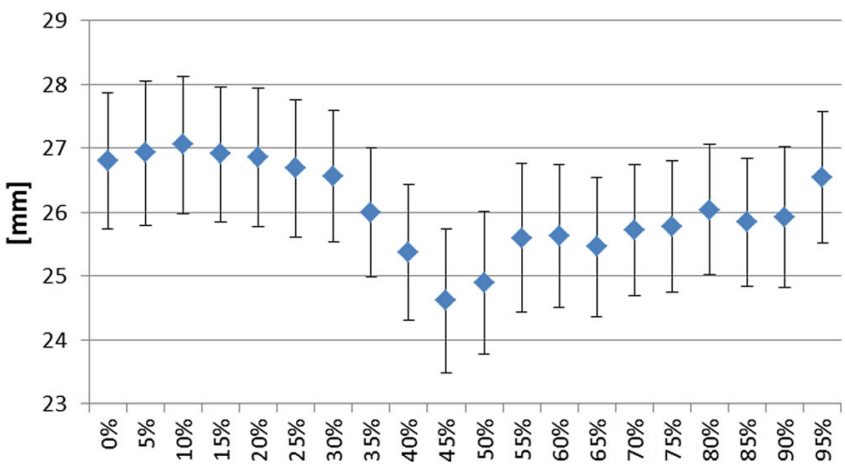

\section{h Relative area}

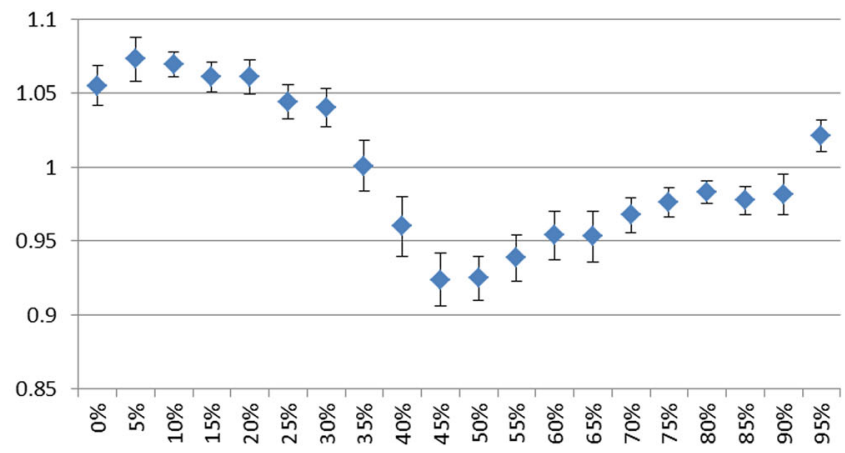

Fig. 4 Mean annulus parameters per cardiac phase. Vertical bars indicate 95\% confidence intervals (CI) 
Table 3 Changes of the annulus parameter values during the cardiac cycle per valve type

\begin{tabular}{|c|c|c|c|c|}
\hline & Max value* & Min value* & $\begin{array}{l}\text { Relative difference }[\%]{ }^{\dagger} \\
( \pm \text { SD of the difference })\end{array}$ & $\begin{array}{l}p \text { value } \neq \\
\text { Min vs Max }\end{array}$ \\
\hline \multicolumn{5}{|c|}{ Maximum diameter [mm] } \\
\hline Sievers type 0 & $30.4 \pm 3.8$ & $28.6 \pm 4.2$ & $2.3 \%( \pm 4.7 \%)$ & 0.207 \\
\hline Sievers type 1 & $30 \pm 4.6$ & $27.9 \pm 4.1$ & $3.9 \%( \pm 5.2 \%)$ & 0.001 \\
\hline Sievers type 2 & $32.3 \pm 2.9$ & $29.8 \pm 3.7$ & $2.7 \%( \pm 3.4 \%)$ & 0.15 \\
\hline \multicolumn{5}{|c|}{ Minimum diameter $[\mathrm{mm}]$} \\
\hline Sievers type 0 & $25.8 \pm 4.1$ & $20.6 \pm 3.9$ & $19.5 \%( \pm 4.5 \%)$ & $<0.001$ \\
\hline Sievers type 1 & $25.5 \pm 3.8$ & $22.2 \pm 3.8$ & $11.6 \%( \pm 7.4 \%)$ & $<0.001$ \\
\hline Sievers type 2 & $29.6 \pm 2.2$ & $23.5 \pm 3.3$ & $19.4 \%( \pm 9.6 \%)$ & 0.028 \\
\hline \multicolumn{5}{|c|}{ Average diameter $[\mathrm{mm}]$} \\
\hline Sievers type 0 & $27.8 \pm 4.2$ & $24.6 \pm 3.7$ & $7.3 \%( \pm 4.7 \%)$ & 0.004 \\
\hline Sievers type 1 & $27.5 \pm 4.1$ & $25 \pm 3.8$ & $7 \%( \pm 3.4 \%)$ & $<0.001$ \\
\hline Sievers type 2 & $30.6 \pm 2.2$ & $27.1 \pm 2.8$ & $7.6 \%( \pm 4.8 \%)$ & 0.028 \\
\hline \multicolumn{5}{|l|}{ Perimeter $[\mathrm{mm}]$} \\
\hline Sievers type 0 & $93.4 \pm 14.3$ & $83.7 \pm 11.8$ & $6 \%( \pm 6 \%)$ & 0.027 \\
\hline Sievers type 1 & $91.8 \pm 14.7$ & $85.8 \pm 13.5$ & $5 \%( \pm 4.9 \%)$ & $<0.001$ \\
\hline Sievers type 2 & $102.5 \pm 8.1$ & $93.7 \pm 11.9$ & $3.5 \%( \pm 5.9 \%)$ & 0.229 \\
\hline \multicolumn{5}{|l|}{ Area $\left[\mathrm{mm}^{2}\right]$} \\
\hline Sievers type 0 & $584 \pm 168$ & $439 \pm 123$ & $17.9 \%( \pm 6.5 \%)$ & 0.001 \\
\hline Sievers type 1 & $574 \pm 173$ & $475 \pm 141$ & $13.2 \%( \pm 6.6 \%)$ & $<0.001$ \\
\hline Sievers type 2 & $691 \pm 117$ & $543 \pm 123$ & $14.2 \%( \pm 10.1 \%)$ & 0.042 \\
\hline \multicolumn{5}{|c|}{ Diameter based on area $[\mathrm{mm}]$} \\
\hline Sievers type 0 & $27 \pm 3.9$ & $23.4 \pm 3.3$ & $9.5 \%( \pm 3.5 \%)$ & $<0.001$ \\
\hline Sievers type 1 & $26.7 \pm 4.0$ & $24.3 \pm 3.7$ & $6.9 \%( \pm 3.6 \%)$ & $<0.001$ \\
\hline Sievers type 2 & $29.6 \pm 2.6$ & $26.1 \pm 3.0$ & $7.5 \%( \pm 5.3 \%)$ & 0.038 \\
\hline \multicolumn{5}{|l|}{ Relative area } \\
\hline Sievers type 0 & $1.1 \pm 0.1$ & $0.9 \pm 0.0$ & $16.4 \%( \pm 6.0 \%)$ & $<0.001$ \\
\hline Sievers type 1 & $1.1 \pm 0.0$ & $0.9 \pm 0.1$ & $13.4 \%( \pm 6.5 \%)$ & $<0.001$ \\
\hline Sievers type 2 & $1.1 \pm 0.1$ & $0.9 \pm 0.1$ & $17.2 \%( \pm 7.5 \%)$ & 0.004 \\
\hline \multicolumn{5}{|l|}{ Asymmetry ratio } \\
\hline Sievers type 0 & $1.4 \pm 0.2$ & $1.1 \pm 0.1$ & $19 \%( \pm 8.6 \%)$ & 0.012 \\
\hline Sievers type 1 & $1.3 \pm 0.1$ & $1.2 \pm 0.1$ & $12.1 \%( \pm 8.8 \%)$ & $<0.001$ \\
\hline Sievers type 2 & $1.3 \pm 0.2$ & $1.1 \pm 0.0$ & $17.6 \%( \pm 11.7 \%)$ & 0.043 \\
\hline
\end{tabular}

*Values calculated based on all data available for each phase; ${ }^{\dagger}$ relative difference compared to the maximum value; ${ }^{\ddagger}$ values calculated based on the number of cases available per comparison of the means; values for relative area and asymmetry ratio were calculated with Wilcoxon signed-ranked test and minimum diameter and $\leq 8.1 \pm 25.9 \mathrm{~mm}^{2}$ and $\leq 18.2 \pm$ $17.9 \mathrm{~mm}^{2}$ for the area respectively.

\section{Discussion}

In this study, we described a standardized way to determine the annulus plane in patients with a BAV and demonstrated that the BAV annulus undergoes significant changes in shape and size during the cardiac cycle. Overall, the annulus showed a larger area with an almost circular shape in systole and a smaller area with an elliptic shape in diastole. Each of the BAV types showed analogous morphological changes. However, type 1 valves had a more elliptic annulus in early systole.

The term BAV includes a spectrum of morphological alterations of the aortic valve that have in common the resulting presence of only two functional cusps. BAV are commonly divided based on the classification system introduced by Sievers et al depending on the presence and number of raphe [1]. A raphe represents the line of fusion between two cusps that are often unequal in size. Different classification systems have been proposed highlighting the variability in morphology and difficulty of categorization of BAV patients [25]. Furthermore, the aortic root is asymmetrically and characteristically enlarged in $58 \%$ of cases [26]. 
Eur Radio (2021) 31:8116-8129

8125
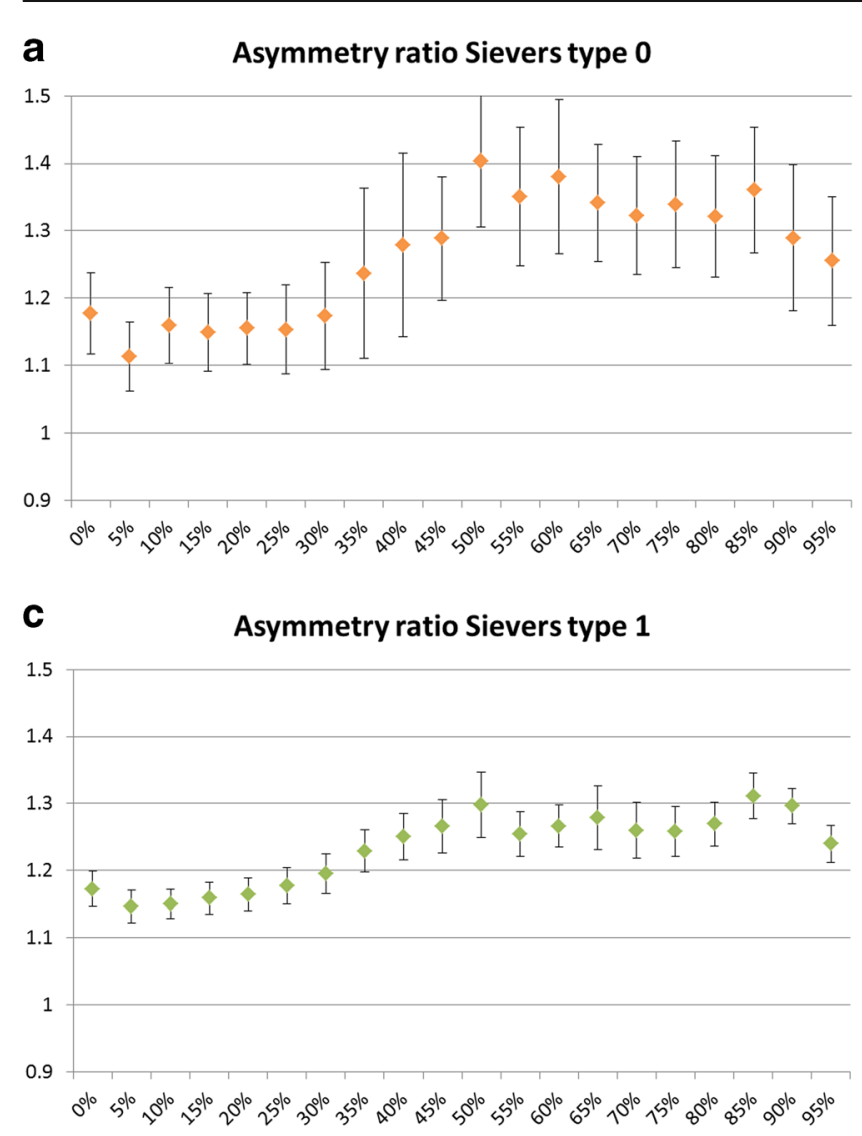

e Asymmetry ratio Silvers type 2
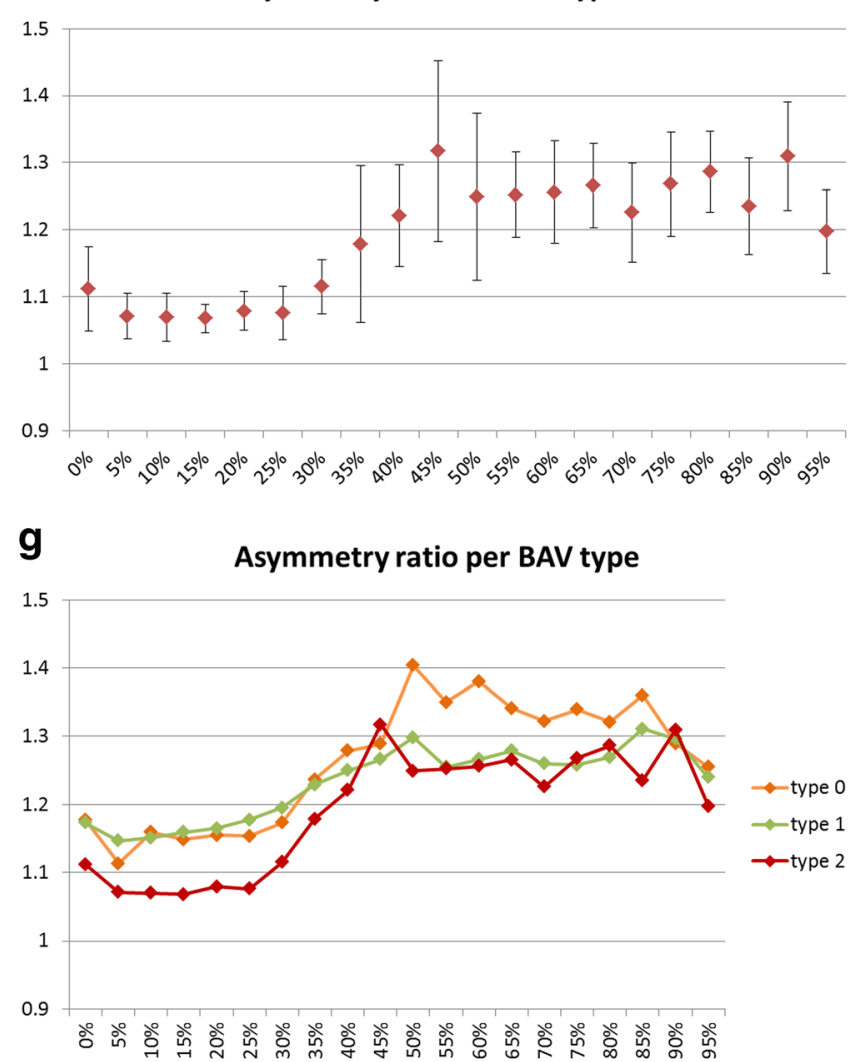
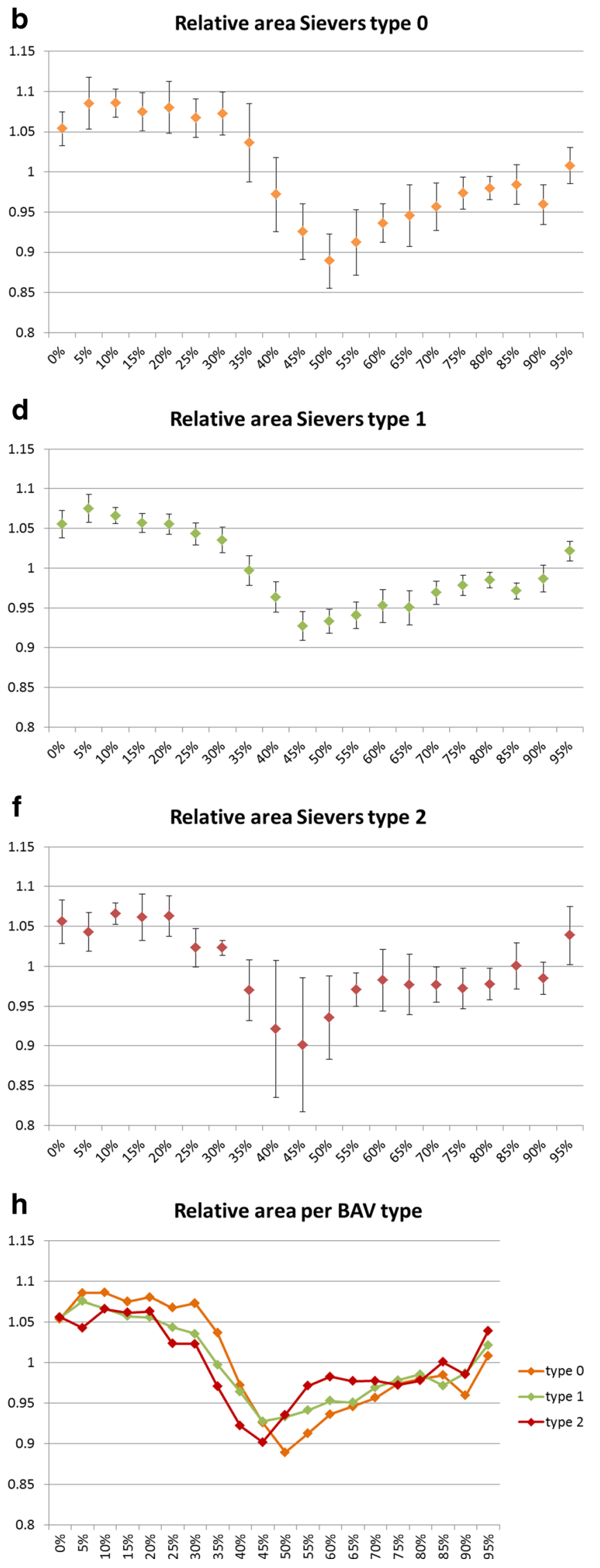

Fig. 5 Mean AR and RA per phase and valve type. Vertical bars indicate 95\% CI

Springer 
Table 4 Classification of scans based on heart rate
Patients' heart rate at the $n$ level of the aortic valve

\begin{tabular}{lr}
\hline$-40-50$ & 6 \\
$-50-60$ & 15 \\
$-60-70$ & 16 \\
$-70-80$ & 10 \\
$-80-90$ & 4 \\
$-90-100$ & 4 \\
$-100-110$ & 2 \\
\hline
\end{tabular}

In the last years, a greater interest in the annulus of BAV patients has emerged, reflecting the increasing resort to TAVI as a therapeutic option in this population. Indeed, the most important entity to be assessed is the ring where the deployed valve will be anchored. This ring, commonly referred to as "annulus," contours the level with the smallest area along the junction between the LVOT and the aortic root but does not correspond to a well-defined anatomical structure [20, 27]. Recent guidelines (intended for patients with TAV) indicate that the annulus should be identified on MPR as the plane passing through the three hinge points of the cusps [18]. Little is known how the modified anatomy of BAV patients affects the definition of the annulus plane [21, 22]. As a consequence, the abovementioned technique based on the three hinge points has been previously applied also to datasets of patients with BAV regardless of valve type [13, 28-30]. We did not find this method feasible for all BAV patients. First, in patients with BAV valve type 0 , there are only two cusps, and therefore, in most cases, only two hinge points (or in few cases two of the three are very close). Since there are infinite planes that pass through two points, more criteria to identify the correct annulus plane had to be introduced as detailed above in the Methods. Contrary to other previously published works, we proposed a combination of multiple criteria for manual identification of the annulus, taking into account the difficulty to visually identify the smallest area as well as the variability of aortic and LVOT characteristics during the cardiac cycle. Furthermore, in patients with BAV type 1 (and possibly type 2 ), one of three cusps is often malformed and/or underdeveloped resulting in a distortion of the normal anatomy of the aortic root. Therefore, the plane passing through the three hinge points might not be representative of the desired plane where to deploy the valve. To the best of our knowledge, we have been the first to acknowledge this intrinsic pitfall for the identification of the annulus plane in BAV type 1 and to propose a feasible solution. Interestingly, this methodology can be directly applied to any cardiac phase and notwithstanding the classification system employed for BAV, since it is based on visual assessment of the number of hinge points and cusps symmetry. With mean differences of $\leq 1 \pm 1.2 \mathrm{~mm}$ for the maximum diameter, inter- and intra-observer variability of the measurements were very good suggesting a good reproducibility of the technique.

Whether reported underexpansion of TAVI prosthesis in BAV patients [28] and increased frequency of paravalvular leakage $[12,31]$ could be due to an overestimation of the annulus size related to the application of the standard methodology for its identification is yet to be confirmed. Also, the potential benefit of our methodology on clinical outcomes has yet to be proven. However, the pressing need to address the issue of correct and specific identification of the annulus in BAV patients seem apparent and of the utmost importance.
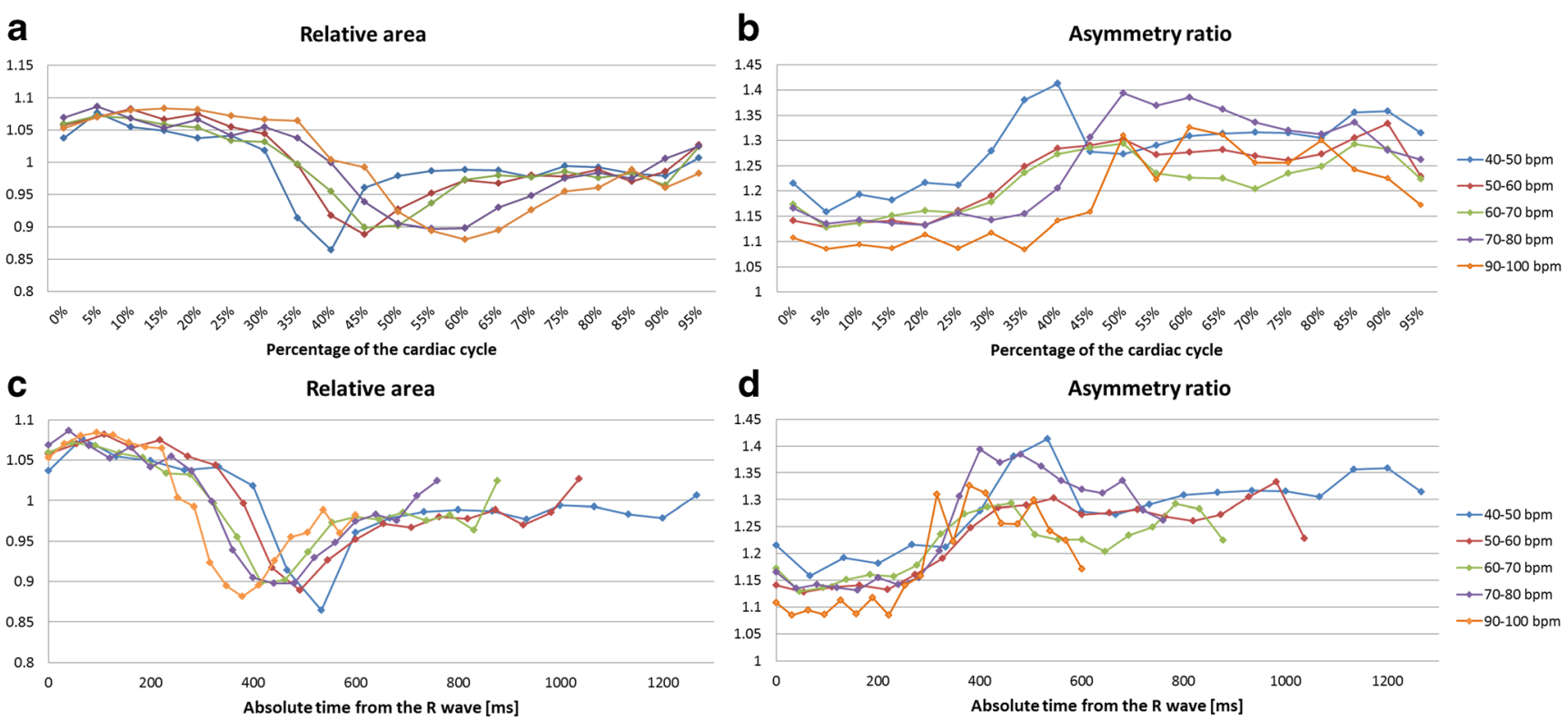

Fig. 6 Asymmetry ratio and relative area in groups with different heart rates during successive phases of the cardiac cycle expressed in terms of percentage (a and $\mathbf{b})$ and absolute time (c and $\mathbf{d})$ 
The application of a specifically tailored methodology such as the one we propose, both in clinical and research settings, will likely improve the standardization of annulus measurements in BAV patients, and thereafter, provide accurate and appropriate sizing data as basis for future guidelines.

In patients with $\mathrm{TAV}$, the annulus has an elliptic shape that undergoes conformational changes during the cardiac cycle as well as significant variation in sizing parameters [7]. In fact, although with some controversy attributable to the better quality and reproducibility of diastolic images [19], literature and guidelines suggest that measurements should be performed on images obtained in systole due to larger annular sizes in this phase $[18,32]$. Our study demonstrated the occurrence of significant changes in sizing parameters during the cardiac cycle in all types of BAV. The RA, minimum diameter, and area showed the most pronounced differences $(13.8 \%$ and $13.7 \%$ ), with all highest values in the phases between 0 and $10 \%$, while the maximum diameter demonstrated the smallest difference $(3.6 \%)$. The relative difference of $7.2 \%$ found for the area-derived diameter corresponded to an absolute difference of $1.9 \mathrm{~mm}$ between systole and diastole. Considering that each prosthetic valve size can be fitted in annuli presenting a range of diameters of $3 \mathrm{~mm}$ at maximum (or $2 \mathrm{~mm}$ depending on the type of valve employed), a $2 \mathrm{~mm}$ difference could imply a different choice of valve size.

Among the reasons that led to consider BAV patients at high risk for TAVI procedures are some characteristics of the aortic annulus such as a more elliptic shape than in patients with TAV. This different conformation has been suggested as the cause for the higher rate of paravalvular regurgitation in BAV patients [10, 14, 33, 34]. With an AR $>1$ at all phases and for all BAV types, but significantly more in early systolic phase for valves type 1, our study demonstrated that the annulus of BAV patients has an elliptic shape. However, the overall and per valve type maximum average AR in our study (maximum AR: $1.3 \pm 0.2$ overall; $1.4 \pm 0.2$ for valves type 0 ; $1.3 \pm 0.1$ for valves type $1 ; 1.3 \pm 0.2$ for valves type 2 ) are comparable to those found in patients with $\operatorname{TAV}[6,8,27]$. Furthermore, compared to already published data, the results of our study confirm that the annulus has larger dimensions in BAV patients (maximum diameter in systole: $30.22 \pm$ $4.48 \mathrm{~mm}$ overall; $30.42 \pm 3.83 \mathrm{~mm}$ for valves type $0 ; 30.04$ $\pm 4.61 \mathrm{~mm}$ for valves type $1 ; 32.34 \pm 2.93 \mathrm{~mm}$ for valves type 2 ) than in patients with TAV [7, 28-30]. Data on TAV show that annulus dynamic characteristics are similar in normal and stenotic valves [6]. Although data from the present study are not sufficient to draw a conclusion in this sense, we expect the same to be true for BAV patients. Considering that these patients often have a dilation of the aorta, we could even expect them to develop a larger annulus later in life.

Hemodynamic characteristics might influence the mechanics of the annulus. Therefore, we investigated the influence of the patients' heart rate at the moment of the CT examination. CT images were reconstructed based on the percentage of the cardiac cycle and therefore the absolute time span between phases depended on the heart rate of the patient at the moment of the acquisition. Our results suggest that, in patients with a higher heart rate, changes in area of the aortic annulus occur at a shorter absolute time, which, however, corresponds to a later phase of the cardiac cycle. Therefore, to ensure that the largest sizing parameters are detected for all heart rates, measurements should be performed in early systolic phase and within $200 \mathrm{~ms}$ from the R wave.

The biggest limitation of the present study is the impossibility to compare planes and measurements obtained with the new method to a reference standard. However, for the annulus, even in TAV patients, there is no reference standard. In fact, measurements performed in operating rooms are subject to limitations including non-physiological conditions and the use of an imprecise measurement tool and, furthermore, values of implanted valve size are influenced by other additional factors. It could be advocated that measurements realized according to the new proposed method should have been compared to those obtained with the standard method. This would be impossible for BAV type 0 for the abovementioned reasons. Moreover, although theoretically possible for asymmetric valves type 1 , it would not yield any significant meaning as the method based on the identification of the three hinge points results in the definition of a plane that does not seem to correspond to the deployment area of the valve. Furthermore, the new method was applied in only 9 cases of type $1 \mathrm{BAV}$. Therefore, our method has to be tested otherwise in a larger cohort of patients. Second, the mean age of the study population was relatively low with a limited number of severely stenotic aortic valves. Obviously, this limits the direct applicability of our findings to patients evaluated for TAVI. However, in TAV patients, similar changes in annulus shape and dimensions were demonstrated for both healthy and stenotic valves [6]. Moreover, due to the relatively limited number of patients per type of BAV valve and, to some extent, to the excluded phases, ANOVA models for repeated measurements were not applicable and comparison between valve types had to be performed per time points. Another limitation that has to be considered is the thickness of the reconstructions $(1.5 \mathrm{~mm})$ which might have influenced the precision of the measurements, although, in our opinion, not so much as to change the results of the study.

To conclude, as assessed based on the proposed refined method for the identification of BAV annulus, in all three BAV types, the annulus has an elliptical shape that undergoes significant size and conformational changes with bigger dimensions in systole and a more elliptical appearance in the diastolic phase. The only exception is type $1 \mathrm{BAV}$ that showed a more elliptical shape in systole as compared to type 2 valves. Our study sheds light on previously unexplored physiology of a multifaceted congenital 
disease. Furthermore, once the proposed method is validated against valve implantation outcomes and in patients with more severe valve dysfunction in further studies, our findings could guide and improve pre-interventional measurements.

Supplementary Information The online version contains supplementary material available at https://doi.org/10.1007/s00330-021-07916-8.

Acknowledgements We thank Sara Baart, Msc, for support in statistical analysis.

Funding This study has received funding by Netherlands Heart Foundation (NHF 2013T093).

\section{Declarations}

Guarantor The scientific guarantor of this publication is Ricardo PJ Budde.

Conflict of interest The authors of this manuscript declare no relationships with any companies whose products or services may be related to the subject matter of the article.

Statistics and biometry Sara Baart kindly provided statistical advice for this manuscript.

Informed consent Written informed consent was obtained from all subjects (patients) in this study.

Ethical approval Institutional Review Board approval was obtained by the Erasmus Medical Center (MEC14-225)

Study subjects or cohorts overlap Some study subjects or cohorts have been previously reported in van den Hoven AT, Mc-Ghie JS, Chelu RG, Duijnhouwer AL, Baggen VJM, Coenen A, et al Transthoracic 3D echocardiographic left heart chamber quantification in patients with bicuspid aortic valve disease. Int J Cardiovasc Imaging. 2017;33(12):1895-9030) Lidia R. Bons, Anthonie L. Duijnhouwer, Sara Boccalini, Allard T. Van Den Hoven, Maureen J. Van Der Vlugt, Raluca G. Chelu, Jackie S. McGhie, Isabella Kardys, Annemien E. Van Den Bosch, Hans-Marc J. Siebelink, Koen Nieman, Alexander Hirsch, Craig S. Broberg, Ricardo P.J. Budde, Jolien W. Roos-Hesselink, Intermodality variation of aortic dimensions: How, where and when to measure the ascending aorta. Ijca (2018), https://doi.org/10.1016/j.ijcard.2018.08.067. However, there is no overlap in objectives or reported data since the aortic annulus was not analyzed in any of these papers.

\section{Methodology \\ - prospective \\ - observational \\ - performed at one institution}

Open Access This article is licensed under a Creative Commons Attribution 4.0 International License, which permits use, sharing, adaptation, distribution and reproduction in any medium or format, as long as you give appropriate credit to the original author(s) and the source, provide a link to the Creative Commons licence, and indicate if changes were made. The images or other third party material in this article are included in the article's Creative Commons licence, unless indicated otherwise in a credit line to the material. If material is not included in the article's Creative Commons licence and your intended use is not permitted by statutory regulation or exceeds the permitted use, you will need to obtain permission directly from the copyright holder. To view a copy of this licence, visit http://creativecommons.org/licenses/by/4.0/.

\section{References}

1. Sievers HH, Schmidtke C (2007) A classification system for the bicuspid aortic valve from 304 surgical specimens. J Thorac Cardiovasc Surg 133:1226-1233

2. Baumgartner H, Bonhoeffer P, De Groot NMS et al (2010) ESC Guidelines for the management of grown-up congenital heart disease (new version 2010). Eur Heart J 31:2915-2957

3. Tutar E, Ekici F, Atalay S, Nacar N (2005) The prevalence of bicuspid aortic valve in newborns by echocardiographic screening. Am Heart. J 150(3):513-515

4. Hoffman JIE, Kaplan S (2002) The incidence of congenital heart disease. J Am Coll Cardiol 39:1890-1900

5. Ward C (2000) Clinical significance of the bicuspid aortic valve. Heart 83:81-85

6. De Heer LM, Budde RPJ, Van Prehn J et al (2012) Pulsatile distention of the nondiseased and stenotic aortic valve annulus: analysis with electrocardiogram-gated computed tomography. Ann Thorac Surg 93:516-522

7. Suchá D, Tuncay V, Prakken NHJ et al (2015) Does the aortic annulus undergo conformational change throughout the cardiac cycle? A systematic review. Eur Heart J Cardiovasc Imaging 16: 1307-1317

8. Hamdan A, Guetta V, Konen E et al (2012) Deformation dynamics and mechanical properties of the aortic annulus by 4-dimensional computed tomography: insights into the functional anatomy of the aortic valve complex and implications for transcatheter aortic valve therapy. J Am Coll Cardiol 59:119-127

9. Smith CR, Leon MB, Mack MJ et al (2011) Transcatheter versus surgical aortic-valve replacement in high-risk patients. N Engl J Med 364:2187-2198

10. Zegdi R, Ciobotaru V, Noghin M et al (2008) Is it reasonable to treat all calcified stenotic aortic valves with a valved stent? Results from a human anatomic study in adults. J Am Coll Cardiol 51:579_ 584

11. Vahanian A, Alfieri O, Andreotti F et al (2012) Guidelines on the management of valvular heart disease (version 2012). Eur Heart J 33:2451-2496

12. Wijesinghe N, Ye J, Rodés-Cabau J et al (2010) Transcatheter aortic valve implantation in patients with bicuspid aortic valve stenosis. JACC Cardiovasc Interv 3:1122-1125

13. Hayashida K, Bouvier E, Lefèvre T et al (2013) Transcatheter aortic valve implantation for patients with severe bicuspid aortic valve stenosis. Circ Cardiovasc Interv 6:284-291

14. Phan K, Wong S, Phan S, Ha H, Qian P, Yan TD (2015) Transcatheter aortic valve implantation (TAVI) in patients with bicuspid aortic valve stenosis - systematic review and meta-analysis. Heart Lung Circ 24:649-659

15. Mylotte D, Lefevre T, Søndergaard L et al (2014) Transcatheter aortic valve replacement in bicuspid aortic valve disease. J Am Coll Cardiol 64:2330-2339

16. van den Hoven AT, Mc-Ghie JS et al (2017) Transthoracic 3D echocardiographic left heart chamber quantification in patients with bicuspid aortic valve disease. Int J Cardiovasc Imaging 33(12): 1895-1903 
17. Bons LR, Anthonie L, Duijnhouwer AL, Boccalini S et al (2018) Intermodality variation of aortic dimensions: how, where and when to measure the ascending aorta. Int J Cardiol 276:230-235

18. Achenbach S, Delgado V, Hausleiter J, Schoenhagen P, Min JK, Leipsic J a. (2012) SCCT expert consensus document on computed tomography imaging before transcatheter aortic valve implantation (TAVI)/transcatheter aortic valve replacement (TAVR). J Cardiovasc Comput Tomogr 6:366-380

19. Kasel AM, Cassese S, Bleiziffer S et al (2013) Standardized imaging for aortic annular sizing: implications for transcatheter valve selection. JACC Cardiovasc Imaging 6:249-262

20. Sievers HH, Hemmer W, Beyersdorf F et al (2012) The everyday used nomenclature of the aortic root components: the tower of babel? Eur J Cardiothorac Surg 41:478-482

21. Frangieh AH, Michel J, Deutsch O et al (2019) Aortic annulus sizing in stenotic bicommissural non-raphe-type bicuspid aortic valves: reconstructing a three-dimensional structure using only two hinge points. Clin Res Cardiol 108:6-15

22. Blanke P, Weir-McCall JR, Achenbach S et al (2019) Computed tomography imaging in the context of transcatheter aortic valve implantation (TAVI)/transcatheter aortic valve replacement (TAVR): an expert consensus document of the Society of Cardiovascular Computed Tomography. JACC Cardiovasc Imaging 12:1-24

23. Queirós S, Dubois C, Morais P et al (2016) Automatic 3D aortic annulus sizing by computed tomography in the planning of transcatheter aortic valve implantation. J Cardiovasc Comput Tomogr. https://doi.org/10.1016/j.jcct.2016.12.004

24. Watanabe Y, Morice MC, Bouvier E et al (2013) Automated 3dimensional aortic annular assessment by multidetector computed tomography in transcatheter aortic valve implantation. JACC Cardiovasc Interv 6:955-964

25. Jilaihawi $\mathrm{H}$, Chen $\mathrm{M}$, Webb J et al (2016) A bicuspid aortic valve imaging classification for the TAVR Era. JACC Cardiovasc Imaging 9:1145-1158

26. Fazel SS, Mallidi HR, Lee RS et al (2008) The aortopathy of bicuspid aortic valve disease has distinctive patterns and usually involves the transverse aortic arch. J Thorac Cardiovasc Surg. https://doi.org/ 10.1016/j.jtcvs.2008.01.022

27. Anderson RH (2000) Clinical anatomy of the aortic root. Heart 84: 670-673

28. Lange R, De Backer O, Hovasse T et al (2019) Bicuspid aortic valve anatomy and relationship with devices: the BAVARD Multicenter Registry. Circ Cardiovasc Interv 12:1-10

29. Son JY, Ko SM, Choi JW et al (2011) Measurement of the ascending aorta diameter in patients with severe bicuspid and tricuspid aortic valve stenosis using dual-source computed tomography coronary angiography. Int J Cardiovasc Imaging 27:61-71

30. Watanabe Y, Chevalier B, Hayashida K et al (2015) Comparison of multislice computed tomography findings between bicuspid and tricuspid aortic valves before and after transcatheter aortic valve implantation. Catheter Cardiovasc Interv 86:323-330

31. Willson AB, Webb JG, Labounty TM et al (2012) 3-dimensional aortic annular assessment by multidetector computed tomography predicts moderate or severe paravalvular regurgitation after transcatheter aortic valve replacement: a multicenter retrospective analysis. J Am Coll Cardiol 59:1287-1294

32. Jurencak T, Turek J, Kietselaer BLJH et al (2015) MDCT evaluation of aortic root and aortic valve prior to TAVI. What is the optimal imaging time point in the cardiac cycle? Eur Radiol 25: 1975-1983

33. Kawamori H, Yoon SH, Chakravarty T et al (2018) Computed tomography characteristics of the aortic valve and the geometry of SAPIEN 3 transcatheter heart valve in patients with bicuspid aortic valve disease. Eur Heart J Cardiovasc Imaging 19:1408-1418

34. Xie X, Shi X, Xun X, Rao L (2016) Efficacy and safety of transcatheter aortic valve implantation for bicuspid aortic valves: a systematic review and meta-analysis. Ann Thorac Cardiovasc Surg 22: 203-215

Publisher's note Springer Nature remains neutral with regard to jurisdictional claims in published maps and institutional affiliations. 